\title{
Skin Corrosion and Irritation Test of Nanoparticles Using Reconstructed Three-Dimensional Human Skin Model, EpiDerm ${ }^{\mathrm{TM}}$
}

\author{
Hyejin Kim', Jonghye Choi', Handule Lee', Juyoung Park', Byung-II Yoon², Seon Mi Jin ${ }^{3}$ and Kwangsik Park \\ ${ }^{1}$ College of Pharmacy, Dongduk Women's University, Seoul, Korea \\ ${ }^{2}$ College of Veterinary Medicine, Kangwon National University, Chuncheon, Korea \\ ${ }^{3}$ College of Medicine, Eulji University, Daejeon, Korea
}

(Received May 24, 2016; Revised August 12, 2016; Accepted August 18, 2016)

\begin{abstract}
Effects of nanoparticles (NPs) on skin corrosion and irritation using three-dimensional human skin models were investigated based on the test guidelines of Organization for Economic Co-operation and Development (OECD TG431 and TG439). EpiDerm ${ }^{\mathrm{TM}}$ skin was incubated with NPs including those harboring iron (FeNPs), aluminum oxide (AlNPs), titanium oxide (TNPs), and silver (AgNPs) for a defined time according to the test guidelines. Cell viabilities of EpiDerm ${ }^{\mathrm{TM}}$ skins were measured by the 3-(4, 5-dimethylthiazol-2-yl)-2.5-diphenyltetrazolium bromide based method. FeNPs, AlNPs, TNPs, and AgNPs were noncorrosive because the viability was more than $50 \%$ after 3 min exposure and more than $15 \%$ after 60 min exposure, which are the non-corrosive criteria. All NPs were also non-irritants, based on viability exceeding 50\% after $60 \mathrm{~min}$ exposure and $42 \mathrm{hr}$ post-incubation. Release of interleukin 1-alpha and histopathological analysis supported the cell viability results. These findings suggest that FeNPs, AINPs, TNPs, and AgNPs are 'non-corrosive' and 'non-irritant' to human skin by a globally harmonized classification system.
\end{abstract}

Key words: Nanoparticles, Skin model, Alternative methods, Skin corrosion, Skin irritation

\section{INTRODUCTION}

Tests of skin corrosion and irritation have long been performed using animals including rabbits based on the test guideline (TG) 404 published by the Organization for Economic Co-operation and Development $(1,2)$. However, the growing ethical recognition of animal welfare has prompted the replacement of in vitro testing of skin corrosion and irritation using animals by alternative methods (3). Regulatory classifications of chemicals with skin corrosion and/or irritation are transitioning from using in vivo data to in vitro data obtained from alternative methods using human skin models (4).

Correspondence to: Kwangsik Park, College of Pharmacy, Dongduk Women's University, \#60, Hwarang-ro, 13-gil, Seongbuk-gu, Seoul 02748, Korea

E-mail: kspark@dongduk.ac.kr

Seon Mi Jin, Eulji Hospital, College of Medicine, Eulji University, 68 Hangeulbiseok-gil, Nowon-gu, Seoul 01830, Korea

E-mail: sunmijin@eulji.ac.kr

This is an Open-Access article distributed under the terms of the Creative Commons Attribution Non-Commercial License (http:// creativecommons.org/licenses/by-nc/3.0) which permits unrestricted non-commercial use, distribution, and reproduction in any medium, provided the original work is properly cited.
Recently, various three-dimensional (3D)-human skin models have become commercially available for the alternative test methods; EpiSkin ${ }^{\mathrm{TM}}$ (EpiSkin Research Institute, Lyon, France), EpiDerm ${ }^{\mathrm{TM}}$ (MatTech Co., Ashland, MA, USA), and SkinEthic ${ }^{\mathrm{TM}}$ (EpiSkin Research Institute) are the most widely used for regulatory purposes. They make use of reconstructed human epidermis obtained from human derived nontransformed epidermal keratinocytes, which closely mimic the histological, morphological, biochemical, and physiological properties of the epidermal layer of human skin $(5,6)$.

Nanoparticles (NPs), defined as small-scale materials less than $100 \mathrm{~nm}$ in at least one dimension, are used in a variety of areas including electronics, cosmetics, pharmaceuticals, and catalysts $(7,8)$. The rapid development of nanotechnology and use of NPs has led to the concern of deleterious human and environmental NP exposure. Hazards of NPs include genotoxicity, reproductive toxicity, and inflammatory responses (7). However, further study is needed to identify human skin related toxicity that includes irritation, sensitization, and corrosion, since human skin can be the first target of NP exposure (9).

Acute dermal irritation and corrosion testing of silver NPs (AgNPs) using rabbits did not show any skin damage including erythema eschar or edema formation for $72 \mathrm{hr}$ after exposure $(10,11)$. However, AgNPs decreased viability and 
increased the inflammatory cytokines levels of interleukin (IL)-1 $\beta$, IL-6, IL-8, and tumor necrosis factor-alpha (TNF$\alpha$ ) in cultured human epidermal keratinocytes (12). Several recent studies of skin corrosion/irritation by NPs utilized in vitro testing with cultured skin-derived cell lines. In vitro data using 3D-human skin models are needed, given the dissimilarity in the data between cell systems and 3Dhuman skin models. Titanium NPs (TNPs) and quantum dot NPs decreased the viability of cultured HaCaT human keratinocytes cell line but did not decrease the viability of a human skin equivalent model $(13,14)$. Nanosilica NPs also caused cell death in cultured human keratinocytes but were not cytotoxic in a human skin equivalent model (15).

In this study, iron NPs (FeNPs), aluminum oxide $\left(\mathrm{Al}_{2} \mathrm{O}_{3}\right)$ NPs (AlNPs), titanium oxide $\left(\mathrm{TiO}_{2}\right) \mathrm{NPs}$ (TNPs), and silver NPs (AgNPs) were investigated using the EpiDerm ${ }^{\mathrm{TM}}$ in vitro 3D-human skin model as an alternative test of skin corrosion and irritation.

\section{MATERIALS AND METHODS}

Materials. FeNPs were purchased from Sigma-Aldrich (St. Louis, MO, USA). As supplied, the NPs were a black powder. Analysis by transmission electron microcopy (TEM) revealed particle size ranging from $35 \sim 45 \mathrm{~nm}$. Purity was $99.5 \%$ based on the trace metals analysis and oxygen content was less than $3 \mathrm{wt} \%$. AlNPs were also purchased from Sigma-Aldrich. Following the product specification supplied by the manufacturer, they were prepared as $20 \mathrm{wt} \%$ suspension in water. TEM analysis showed a particle size range of 30 60 nm. AgNPs (Sigma-Aldrich) were prepared as a dark grey powder and contained polyvinyl pyrrolidone as dispersant. Purity was $99.5 \%$ based on the trace metals analysis and TEM-determined particle size was less than $100 \mathrm{~nm}$. TNPs (P-25, $21 \mathrm{~nm}$ diameter size; Aeroxide $\left.{ }^{\circledR}\right)$ were kindly provided by Evonik Industry (Ham, France) as part of an OECD program for nanomaterial assessments. P-25 was supplied as a fine white powder with a hydrophilic character due to surface hydroxyl groups (ca. five $\mathrm{OH}$ groups per $\mathrm{nm}^{2}$ ). It consists of aggregated primary particles. The aggregates were several hundred $\mathrm{nm}$ in size. Mean diameter of the primary particles was approximately $20 \mathrm{~nm}$.

The EpiDerm ${ }^{\mathrm{TM}}$ consists of normal human-derived keratinocytes, multiple viable cell layers, and functional stratum corneum. It is an OECD-validated skin model. EpiDerm ${ }^{\mathrm{TM}}$ samples were provided on specially prepared cell culture inserts prepared by the manufacturer. Immunoassay kit for human IL-1 $\alpha$ was purchased from R\&D Systems (Minneapolis, MN, USA).

EpiDerm $^{T M}$ and NP exposure. For the in vitro skin corrosion test, the EpiDerm ${ }^{\mathrm{TM}}$ inserts were transferred to wells of 6-well plates containing $0.9 \mathrm{~mL}$ Dulbecco's Modified Eagle's Medium (DMEM) and pre-incubated in a $5 \% \mathrm{CO}_{2}$ incubator overnight according to the supplier's protocol. After pre-incubation, the EpiDerm ${ }^{\mathrm{TM}}$ inserts were exposed to FeNPs, AlNPs, AgNPs, and TNPs for 3 min, and separately for $60 \mathrm{~min}$ based on OECD test guideline (TG) 431 . As specified by the TG, the epidermis surface was moistened with deionized water if the $25 \mathrm{mg}$ of test material was a powder (FeNPs, AgNPs, and TNPs) to improve contact. AlNPs were supplied as a liquid; $50 \mu \mathrm{L}$ was directly applied to the epithelial insert. $\mathrm{KOH}(8 \mathrm{~N})$ was used as positive chemical and phosphate buffred saline (PBS) was the normal control. Three replicates of EpiDerm ${ }^{\mathrm{TM}}$ were used for each experiment. After exposure, the test materials were removed from the exposed skin models by repeated rinsing with PBS, and they were transferred to the new plates for viability testing as described below.

For the OECD TG439 in vitro irritation test, skin models were exposed to NPs for 60 min. Sodium dodecyl sulfate ( $5 \%$ solution) was used as the positive chemical. Following exposure, test materials were removed and repeatedly rinsed with PBS. Post-incubation of the exposed skin models was done in fresh medium for $42 \mathrm{hrs}$ prior to the viability assay.

Viability assay. For viability testing, the insert was transferred to a well of a 24-well plate. 3-(4,5-Dimethylthiazol-2-yl)-2,5-diphenyltetrazolium bromide (MTT; $300 \mu \mathrm{L}$ of a $0.3 \mathrm{mg} / \mathrm{mL}$ solution) was added to each well. Samples were incubated in a $5 \% \mathrm{CO}_{2}$ incubator for $3 \mathrm{hrs}$ and insoluble formazan products of MTT were extracted by $2 \mathrm{~mL}$ isopropanol that was added to each well. A $200 \mu \mathrm{L}$ portion of the isopropanol extract was transferred to a well of a 96-well plate and the optical density (OD) was measured at $570 \mathrm{~nm}$.

IL-1 $\alpha$ assay and histopathology. During the $42 \mathrm{hr}$ post-incubation period of the skin irritation test, the medium was replaced with fresh medium at $24 \mathrm{hr}$. The replaced medium was kept for the IL-1 $\alpha$ assay. After coating wells of a 96-well plate with capture antibody, $100 \mu \mathrm{L}$ aliquots of the medium obtained at $24 \mathrm{hr}$ were added to wells and incubated for $2 \mathrm{hr}$ at room temperature. After washing the wells, detection antibody was added and the plate was incubated at room temperature for $2 \mathrm{hr}$. Following the washing steps, streptavidin-horse radish peroxidase-conjugated secondary antibody was added to the wells and the plate was incubated for $20 \mathrm{~min}$. After washing, substrate solution was added and incubated for $20 \mathrm{~min}$. After incubation, the reaction was stopped by adding $2 \mathrm{~N}$ sulfuric acid. The OD was measured at $450 \mathrm{~nm}$ using a microplate reader. The concentration was calculated using a standard curve.

For the histopathological examination, EpiDerm ${ }^{\mathrm{TM}}$ was taken and prefixed in $10 \%(\mathrm{v} / \mathrm{v})$ neutral buffered formaldehyde. The fixed tissues were trimmed, dehydrated, embedded in paraffin, sectioned, mounted on glass slides, stained with hematoxylin and eosin, and examined by light microscopy. 
Interpretation of in vitro test results. According to OECD TG431, the test material is considered to be corrosive to the skin if the viability is $<50 \%$ after $3 \mathrm{~min}$ exposure. Although the viability after $3 \mathrm{~min}$ exposure is $\geq 50 \%$, it is corrosive if the viability is $<15 \%$ after $60 \mathrm{~min}$ exposure. The material is non-corrosive if the viability is $\geq 50 \%$ after $3 \mathrm{~min}$ exposure and $\geq 15 \%$ after $60 \mathrm{~min}$ exposure. Regarding the irritation test, the test material is considered to be irritant to skin if the tissue viability after exposure/ post-incubation is $\leq 50 \%$. It is a non-irritant if the viability is $>50 \%$ in accordance with OECD TG439.

\section{RESULTS}

In vitro skin corrosion test. In the corrosion test, cell viability of EpiDerm ${ }^{\mathrm{TM}}$ exposed to the positive chemical $8 \mathrm{~N}$ $\mathrm{KOH}$ for $3 \mathrm{~min}$ was decreased to $9.8 \%$. However, the mean cell viability of EpiDerm ${ }^{\mathrm{TM}}$ treated with NPs for 3 min was $87.5 \sim 96.3 \%$. When the exposure time was increased to 60 min, viability decreased to $49.8 \%$ in FeNP treated samples, 58.7\% in AgNP treated samples, 97.7\% in AlNP treated samples, and $85.3 \%$ in TNP treated samples (Table 1). The decreased viability by FeNPs and AgNPs was not indicative of skin corrosive behavior. Based on OECD TG431, all the tested NPs were non-corrosive because the viability exceeded $50 \%$ and $15 \%$ after 3 and 60 min exposure, respectively.

In vitro skin irritation test. In the irritation test, EpiDerm $^{\mathrm{TM}}$ cells were exposed to NPs and 5\% sodium dodecyl sulfate (SDS) as the positive chemical for $60 \mathrm{~min}$. The treated materials were washed out completely and the skins of EpiDerm ${ }^{\mathrm{TM}}$ were post-incubated for $42 \mathrm{hr}$. EpiDerm ${ }^{\mathrm{TM}}$ cell viability was $8.4 \pm 0.2 \%$ in the $5 \%$ SDS-treated group after $42 \mathrm{hr}$. However, the viabilities of the NP-treated groups were not as comparably low: $80.9 \pm 17.2 \%$ with FeNP treatment, $71.6 \pm 31.0 \%$ with AlNP treatment, $94.4 \pm 2.5 \%$ with TNP treatment, and $81.3 \pm 1.8 \%$ with AgNP treatment (Table 2). Based on OECD TG439, all the NP preparations were non-irritants because the viability exceeded $50 \%$ after $60 \mathrm{~min}$ exposure and $42 \mathrm{hr}$ incubation.

IL-1 $\alpha$ assay and histopathology. IL- $1 \alpha$ is a marker of pro-inflammation. IL- $1 \alpha$ in irritated skin was increased to $769.2 \pm 7.6 \mathrm{pg} / \mathrm{mL}$ in the culture medium obtained from the $5 \%$ SDS treated group (Table 3), while the level of IL- $1 \alpha$ in the PBS control was $189.8 \pm 9.3 \mathrm{pg} / \mathrm{mL}$. However, no significant increase was observed for any of the NP preparations, supporting their non-irritant nature. No histopathological changes were evident in EpiDerm ${ }^{\mathrm{TM}}$ skin treated with NPs (Fig. 1). NP treatment did not damage the keratin, granular, prickle cell, and basal layers (Fig. 1A-1D). PBS treatment was also not damaging (Fig. 1E). The keratin layer was characteristically dense and sufficient granules were evident in the granular layer. However, serious corrosion and destruction of skin structure was observed upon treatment with $8 \mathrm{~N} \mathrm{KOH}$, with corrosion and removal of cell layers evident (Fig. 1F).

\section{DISCUSSION}

Industrial and consumer uses of engineered NPs have

Table 1. Cell viabilities of $3 D$ human skin model EpiDerm ${ }^{\mathrm{TM}}$ in skin corrosion test

\begin{tabular}{cccccc}
\hline \hline Cell viability (\%) & \multicolumn{1}{c}{ FeNPs } & AlNPs & TNPs & AgNPs & KOH \\
\hline $3 \mathrm{~min}$ & $87.5 \pm 4.3^{* * *}$ & $90.9 \pm 4.6^{* *}$ & $96.3 \pm 2.4$ & $89.0 \pm 1.9^{* * *}$ & $9.8 \pm 1.6^{* * *}$ \\
$60 \mathrm{~min}$ & $49.8 \pm 28.6^{* * *}$ & $97.9 \pm 5.8$ & $85.3 \pm 3.9$ & $58.7 \pm 43.1^{* *}$ & $8.3 \pm 0.7^{* * *}$ \\
\hline
\end{tabular}

EpiDerm ${ }^{\mathrm{TM}}$ were treated with nanoparticles for $3 \mathrm{~min}$ and $60 \mathrm{~min}$, respectively and MTT assay was performed. Data were expressed as the mean \pm S.D. $(\mathrm{N}=3)$. Significant differences, ${ }^{* *} p<0.01,{ }^{* * *} p<0.001$.

Table 2. Cell viability of 3D human skin model in skin irritation test

\begin{tabular}{cccccc}
\hline \hline & FeNPs & AlNPs & TNPs & AgNPs & SDS \\
\hline Cell viability (\%) & $80.9 \pm 17.2$ & $71.6 \pm 31.0^{* *}$ & $94.4 \pm 2.5$ & $81.3 \pm 1.8$ & $8.4 \pm 0.2^{* * *}$ \\
\hline
\end{tabular}

EpiDerm $^{\mathrm{TM}}$ were treated with nanoparticles for $60 \mathrm{~min}$ and post-incubated for $42 \mathrm{hr}$ and MTT assay was performed. Data were expressed as the mean \pm S.D. $(\mathrm{N}=3)$. Significant differences, ${ }^{* *} p<0.01,{ }^{* * *} p<0.001$

Table 3. IL-1 $\alpha$ concentration in the media of cultured 3D human skin model EpiDerm ${ }^{\mathrm{TM}}$ in skin irritation test

\begin{tabular}{lcccccc}
\hline \hline & FeNPs & AlNPs & TNPs & AgNPs & SDS & PBS \\
\hline $\mathrm{IL}-1 \alpha(\mathrm{pg} / \mathrm{mL})$ & $85.4 \pm 4.6$ & $97.4 \pm 6.3$ & $67.7 \pm 0.7$ & $131.1 \pm 4.9$ & $769.2 \pm 7.6$ & $189.8 \pm 9.3$ \\
\hline EpiDerm $^{\mathrm{TM}}$ were treated with nanoparticles for 60 min and post-incubated for $42 \mathrm{hr}$. During the $42 \mathrm{hr}$ post-incubation period of skin irrita- \\
tion test, the media were replaced by fresh media at $24 \mathrm{hr}$ and IL-1 $\alpha$ was measured in the replaced media. Data were expressed as the \\
mean \pm S.D. $(\mathrm{N}=3)$.
\end{tabular}



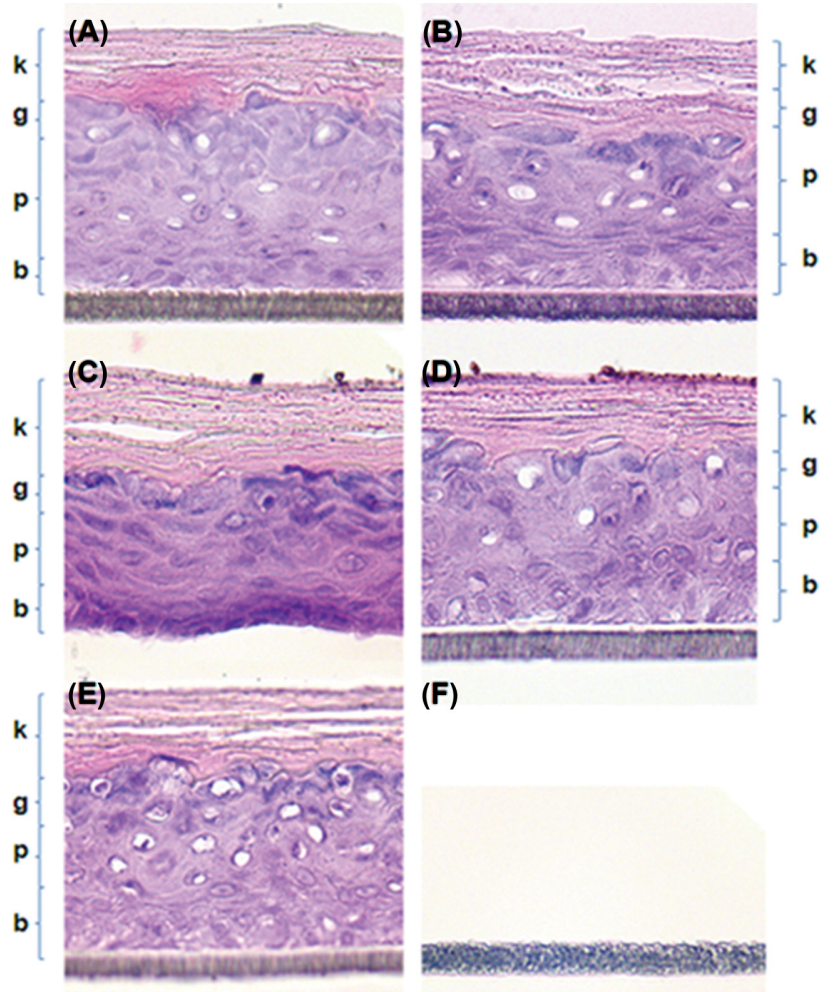

(F)

Fig. 1. Histopathological examination of the EpiDerm ${ }^{\mathrm{TM}} 3 \mathrm{D}$ human skin model by hematoxylin and eosin ( $H$ \& E) staining $(\times 400)$. Histopathologically significant changes were not observed in the NP treated groups (A) (D) and normal control groups (E). Keratin layer $(k)$, granular layer $(g)$, prickle cell layer $(p)$, and basal layer (b) were obvious in NP treated groups (A) (D) as well as in the normal control group (E). However, skin layers seem to be completely corroded out by treatment with $8 \mathrm{~N} \mathrm{KOH}$ (F). (A) FeNPs treated group, (B) AlNPs treated group, (C) TNPs treated group, (D) AgNPs treated group, (E) Normal control group, (F) $8 \mathrm{~N} \mathrm{KOH}$ treated group.

increased to include applications as catalysts, and in the oil, textile, and cosmetic industries, and in medicine $(16,17)$. As the uses of NPs have grown, the risks of environmental and human toxicity have also increased. Several studies that investigated NP-related hazards reported inflammatory responses, immune defects, genotoxicity, reproductive toxicity, developmental toxicity, and other related adverse effects, which may be caused by NP-mediated generation of reactive oxygen species (ROS) $(18,19)$.

TNPs have a variety of applications. Most commonly they are used in pigments, paints, and cosmetics. The generation of ROS and cytotoxicity has been described when TNPs were added to cultured cells. However, information concerning TNP-related skin irritation is still limited. Ultraviolet and Visible-A (UV-A) irradiation of TNPs can induce significant cell damage of human skin keratinocytes. The damage is mediated by lipid and protein peroxidation. This phototoxicity is reportedly mediated by ROS generated during UV-A irradiation (20). However, another study reported that TNPs did not induce phototoxicity, acute cutaneous irritation, or skin sensitization in a human skin model (13). The penetration of nanomaterials in sunscreen formulations was observed when the mixtures of TNPs and zinc NPs were applied to UV-B damaged skin, but no transdermal absorption was detected (21). NPs made from iron or iron oxide, have also reportedly mediated ROS-linked redox reactions. A recent study showed that alveolar macrophages exposed to FeNPs increased their production of ROS, nitric oxide, and cytokines (22). Exposure to FeNPs can alter cell morphology, cytoskeleton of cells, and cell motility (23). Genotoxic effects of FeNPs are mainly influenced by direct interaction with leached iron ions from FeNPs or various indirect factors, such as excessive production of ROS (24). However, information concerning FeNP-related skin toxicity is scant, as is information on the effects of AlNPs and AgNPs on skin corrosion and skin irritation. In the present study, we sought to find direct evidence of an effect of NPs on skin corrosion and skin irritation using a 3D-human skin model, and explored whether the skin model would be good for regulatory purposes.

As specified in the OECD TG431 and TG439, test materials were directly applied to the skin model submerged into culture medium. Slight aggregation occurred when NPs were applied to the skin surface. The EpiDerm ${ }^{\mathrm{TM}}$ was completely destroyed by $8 \mathrm{~N} \mathrm{KOH}$, with most cells killed after only a 3 -min exposure (Table 1 ). Cell viability was not further decreased when the exposure time was increased to $60 \mathrm{~min}$. So, $8 \mathrm{~N} \mathrm{KOH}$ was toxic enough to cause corrosion in a very short time. EpiDerm ${ }^{\mathrm{TM}}$ viability decreased with FeNP, TNP, and AgNP treatment as the exposure time increased from $3 \mathrm{~min}$ to $60 \mathrm{~min}$, while AlNPs did not decrease the viability of EpiDerm ${ }^{\mathrm{TM}}$. A marked difference in EpiDerm ${ }^{\mathrm{TM}}$ viability was evident upon FeNP treatments; viability decreased from $87.5 \pm 4.3 \%$ at 3 min exposure to $49.8 \pm 28.6 \%$ at $60 \mathrm{~min}$ exposure. According to OECD TG431, EpiDerm ${ }^{\mathrm{TM}}$ viability is judged to be non-corrosive when viability is $\geq 50 \%$ after 3 min exposure and $>15 \%$ at $60 \mathrm{~min}$ exposure. Thus, all NPs tested in this study were non-corrosive.

Concerning skin irritation, viability upon treatment with $5 \% \mathrm{SDS}$ as the positive chemical was decreased to $8.4 \pm$ $0.2 \%$, while the viabilities upon treatment with all NP preparations exceeded the $50 \%$ limit value for skin irritation. The difference between the skin corrosion and irritation tests was the 42-hr post-incubation after the $60 \mathrm{~min}$ exposure. During the post-incubation period, reversibly damaged cells can be restored to be viable. Viabilities of the NP preparations, except for the AlNP-treated group, were higher in the skin irritation test compared to the skin corrosion test. Although the viability of AlNPs in the skin irritation test was lower than in the skin corrosion test, it was in 
the range of standard deviation. As shown in Table 1 and Table 2, the test nanoparticles were not corrosive and irritant based on the OECD TG criteria. However, they showed week cytotoxicity to EpiDerm ${ }^{\mathrm{TM}}$. It means that NPs may cause adverse effects in skin cells when they are exposed to NPs. Further studies on the toxic mechanism of NPs in skin cell including cell death mechanism will be necessary.

IL-1 $\alpha$ production was not elevated in the medium of each NP-treated group compared to the positive SDS control (Table 3). Although the levels of IL-1 $\alpha$ in the tested groups are not same level but lower than the PBS control group, it does not distract the conclusion for non-irritation/non-corrosive. None of the NP preparations induced cell death or release of IL- $1 \alpha$ in levels indicative of skin irritation. The non-corrosive nature of the NPs was supported by the histopathology observations that indicated lack of damage (Fig. 1). $\mathrm{KOH}$, which is corrosive, completely damaged the skin structure.

In summary, FeNPs, AlNPs, TNPs, and AgNPs were tested for skin corrosion and irritation in vitro based on the OECD TG431 and TG439. The NPs were non-corrosive and nonirritant.

\section{ACKNOWLEDGMENTS}

This work was supported by National Institute of Environmental Research, Korea.

\section{REFERENCES}

1. OECD (2002) Test No. 404: Acute dermal irritation/corrosion, OECD guidelines for the testing of chemicals, section 4. Available from: http://dx.doi.org/10.1787/9789264070622-en.

2. OECD (2015) Test No. 404: Acute dermal irritation/corrosion, OECD guidelines for the testing of chemicals, section 4 . Available from: http://dx.doi.org/10.1787/9789264242678-en.

3. Murthy, P.B., Kishore, A.S. and Surekha, P. (2012) Assessment of in vitro skin irritation potential of nanoparticles: RHE model. Methods Mol. Biol., 926, 219-234.

4. Basketter, D., Jírova, D. and Kandárová, H. (2012) Review of skin irritation/corrosion hazards on the basis of human data: A regulatory perspective. Interdiscip. Toxicol., 5, 98-104.

5. OECD (2015) Test No. 431: In vitro skin corrosion: reconstructed human epidermis (RHE) test method, OECD guidelines for the testing of chemicals, section 4. Available from: http://dx.doi.org/10.1787/9789264242753-en.

6. OECD (2015) Test No. 439: In vitro skin irritation: reconstructed human epidermis test method, OECD guidelines for the testing of chemicals, section 4. Available from: http:// dx.doi.org/10.1787/9789264242845-en.

7. Hofmann-Amtenbrink, M., Grainger, D.W. and Hofmann, H. (2015) Nanoparticles in medicine: current challenges facing inorganic nanoparticle toxicity assessments and standardizations. Nanomedicine, 11, 1689-1694.

8. Hussain, I., Singh, N.B., Singh, A., Singh, H. and Singh, S.C. (2016) Green synthesis of nanoparticles and its potential application. Biotechnol. Lett., 38, 545-560.

9. Franken, A., Eloff, F.C., Du Plessis, J. and Du Plessis, J.L. (2015) In vitro permeation of metals through human skin: a review and recommendations. Chem. Res. Toxicol., 28, 22372249.

10. Kim, J.S., Song, K.S., Sung, J.H., Ryu, H.R., Choi, B.G., Cho, H.S., Lee, J.K. and Yu, I.J. (2013) Genotoxicity, acute oral and dermal toxicity, eye and dermalirritation and corrosion and skin sensitisation evaluation of silvernanoparticles. Nanotoxicology, 7, 953-960.

11. Paladini, F., Sannino, A. and Pollini, M. (2014) In vivo testing of silver treated fibers for the evaluation of skin irritation effect and hypoallergenicity. J. Biomed. Mater. Res. Part B Appl. Biomater, 102, 1031-1037.

12. Samberg, M.E., Oldenburg, S.J. and Monteiro-Riviere, N.A. (2010) Evaluation of silver nanoparticle toxicity in skin in vivo and keratinocytes in vitro. Environ. Health Perspect., 118, 407-413.

13. Park, Y.H., Jeong, S.H., Yi, S.M., Choi, B.H., Kim, Y.R., Kim, I.K., Kim, M.K. and Son, S.W. (2011) Analysis for the potential of polystyrene and $\mathrm{TiO} 2$ nanoparticles to induce skin irritation, phototoxicity, and sensitization. Toxicol. In Vitro, 25, 1863-1869.

14. Jeong, S.H., Park, Y.H., Choi, B.H., Kim, J.H., Sohn, K.H., Park, K.L., Kim, M.K. and Son, S.W. (2010) Assessment of the skin irritation potential of quantum dot nanoparticles using a human skinequivalent model. J. Dermatol. Sci., 59, 147-148.

15. Park, Y.H., Kim, J.N., Jeong, S.H., Choi, J.E., Lee, S.H., Choi, B.H., Lee, J.P., Sohn, K.H., Park, K.L., Kim, M.K. and Son, S.W. (2010) Assessment of dermal toxicity of nanosilica using cultured keratinocytes, a human skin equivalent model and an in vivo model. Toxicology, 267, 178-181.

16. Contado, C. (2015) Nanomaterials in consumer products: a challenging analytical problem. Front. Chem., 3, 48.

17. Stark, W.J., Stoessel, P.R., Wohlleben, W. and Hafner, A. (2015) Industrial applications of nanoparticles. Chem. Soc. Rev., 44, 5793-5805.

18. Khalili Fard, J., Jafari, S. and Eghbal, M.A. (2015) A review of molecular mechanisms involved in toxicity of nanoparticles. Adv. Pharm. Bull., 5, 447-454.

19. Chen, G., Vijver, M.G. and Peijnenburg, W.J. (2015) Summary and analysis of the currently existing literature data on metal-based nanoparticles published for selected aquatic organisms: applicability for toxicity prediction by (Q)SARs. Altern. Lab. Anim., 43, 221-240.

20. Yin, J.J., Liu, J., Ehrenshaft, M., Roberts, J.E., Fu, P.P., Mason, R.P. and Zhao, B. (2012) Phototoxicity of nano titanium dioxides in $\mathrm{HaCaT}$ keratinocytes--generation of reactive oxygen species and cell damage. Toxicol. Appl. Pharmacol., 263, 81-88.

21. Monteiro-Riviere, N.A., Wiench, K., Landsiedel, R., Schulte, S., Inman, A.O. and Riviere, J.E. (2011) Safety evaluation of sunscreen formulations containing titanium dioxide and zinc oxide nanoparticles in UVB sunburned skin: an in vitro and in vivo study. Toxicol. Sci., 123, 264-280.

22. Ahamed, M., Alhadlaq, H.A., Alam, J., Khan, M.A., Ali, D. and Alarafi, S. (2013) Iron oxide nanoparticle-induced oxidative stress and genotoxicity in human skin epithelial and lung epithelial cell lines. Curr. Pharm. Des., 19, 6681-6690. 
23. Astanina, K., Simon, Y., Cavelius, C., Petry, S., Kraegeloh, A. and Kiemer A.K. (2014) Superparamagnetic iron oxide nanoparticles impair endothelial integrity and inhibit nitric oxide production. Acta Biomater., 10, 4896-4911.

24. Mesárošová, M., Kozics, K., Bábelová, A., Regendová, E.,
Pastorek, M., Vnuková, D., Buliaková, B., Rázga, F. and Gábelová, A. (2014) The role of reactive oxygen species in the genotoxicity of surface-modified magnetite nanoparticles. Toxicol. Lett., 226, 303-313. 